\title{
ESTUDIO BIOMÉTRICO Y MORFOLÓGICO DE LOS HUEVOS DE PALAEMONETES VARZANS LEACH DE DOS LOCALIDADES DEL SUROESTE ESPAÑOL
}

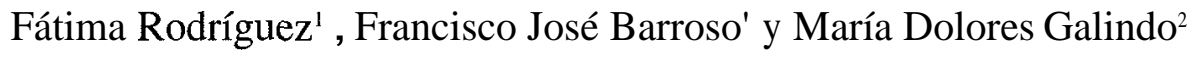 \\ ${ }^{1}$ Departamento de Fisiología y Biología Animal. Apdo. 1095. E-41080- SEVILLA. \\ ${ }^{2}$ Departamento de Biología Vegetal y Ecología. Apdo. 1095. E-41080- SEVILLA. (Autor al que dirigir la correspondencia)
}

Palabras clave: Palaemonetes varians, huevos, biometría, morfología, estrategia reproductiva.

\author{
ABSTRACT \\ BIOMETRIC AND MORPHOLOGIC STUDY OF EGGS OF PALAEMONETES VARIANS LEACH FROM TWO \\ LOCALITIES OF THE SPANISH SOUTHWEST.
}

Two natural populations of Palaemonetes varians LEACH proceeding from two localities of the Spanish Southwest and subjected to different salinities have been compared, on account of their reproduction abilities. Five embryonic developmental stages have been defined. Although no differences in biometric measures of eggs from both localities were found, there were differences in reproductive strategies. The influence of temperature on the survival of progenitors has been studied in the laboratory, debating the adaptative significance of the results.

\section{INTRODUCCIÓN}

La talla del huevo es un factor determinante para la supervivencia y el crecimiento del recién nacido, particularmente en aquellos organismos acuáticos cuyos progenitores no alimentan a sus crías. Debido a la limitación de la materia o energía reproductiva en el progenitor, la talla óptima del huevo combinada con el número de éstos maximiza el número de recién nacidos que sobrevive hasta la madurez (MASHIKO, 1982 y 1983). HELDT (1953) encuentra que dependiendo de la salinidad, distintos huevos y larvas conducen a adultos idénticos. Se han encontrado diferencias en el tamaño y número de huevos para Palaemon paucidens y Macrobrachium nipponense en poblaciones aisladas reproductivamente sometidas a condiciones ambientales cualitativamente distintas (MASHIKO, 1982 y 1983) y para las especies simpátricas Palaemonetes vulgaris y Palaemonetes pugio dependiendo del hábitat, como respuesta contra la predación (YAN, 1987).

Palaemonetes varians LEACH, 1814 habita en aguas salobres estuarinas, marismas y charcas intermareales (ANTHEUNISSE et al. ,1977; YUFERA y RODRIGUEZ, 1985), siendo un organismo muy euríhalino (SOLLAUD, 1939). En el Suroeste de España se han encontrado poblaciones de esta especie habitando zonas continentales de diferente salinidad. el objetivo de este estudio es comprobar si existen diferencias en los parámetros reproductivos anteriormente citados, como respuesta a una estrategia adaptativa. Para ello se han considerado hembras grávidas de dos poblaciones, una procedente de una marisma mareal y otra de una marisma de carácter fluvial; estableciéndose cinco estadíos en el desarrollo embrionario de los huevos a lo largo del proceso de incubación-eclosión. Finalmente, se ha observado experimentalmente la influencia del aumento de la temperatura en el proceso de incubación de huevos y supervivencia del progenitor.

\section{MATERIAL Y MÉTODOS}

El material zoológico utilizado para los estudios biométrico y morfológico ha sido recolectado, durante varios meses de 1990, de dos zonas que presentan distinto régimen de salinidad: marismas de Ayamonte (Huelva) capturado en aguas con $32 \mathrm{~g} / \mathrm{l}$ de salinidad; y curso bajo del río Guadalquivir en las marismas de Villafranco (Sevilla) cuya salinidad oscila entre 5 y $35 \mathrm{~g} / \mathrm{l}$, en aguas de $6 \mathrm{~g} / \mathrm{l}$, en este caso.

Para la caracterización morfológica de las diferentes etapas de desarrollo del embrión durante el proceso de

Limnética, 9: 67-72 (1993)

O Asociación Española de Limnología, Madrid. Spain 
incubación-eclosión se han descrito 5 estadíos de desarrollo en función de los cambios morfológicos ocurridos durante la embriogénesis. El estadío 5 se ha subdividido en dos respecto a la biometría debido a los cambios observados justo en el momento previo a la eclosión.

Del conjunto de ejemplares capturados se seleccionó un grupo de hembras grávidas, las cuales fueron mantenidas en acuarios con agua de su lugar de procedencia, que fue renovada periódicamente. De las hembras ovígeras se determinaron, tomando tres réplicas por cada estadío de desarrollo, los siguientes parámetros: peso de la hembra con huevos, peso de la hembra sin huevos, peso de la puesta, número de huevos y longitud corporal. De cada uno de los ejemplares se extrajeron 10 huevos a los cuales se midió la longitud de los ejes mayor y menor. El peso fresco se ha determinado con una precisión de milésimas de gramo, después de mantener el material durante 5 minutos en reposo sobre papel secante. El peso de la puesta se ha calculado por diferencia entre el peso de los ejemplares con y sin huevos (CALDENTEY et al., 1988), y la longitud corporal se ha considerado como la distancia existente entre el borde posterior del télson y el extremo anterior del ojo (MASHIKO, 1983). La longitud de los ejes de los huevos (L y 1) se determinó mediante un objetivo micrométrico, con una precisión de $0,05 \mathrm{~mm}$. El volumen de los huevos se ha determinado a partir de la longitud de los ejes utilizando la fórmula del volumen de un elipsoide (MASHIKO, 1982; RAMONELL et al., 1987); calculándose también la superficie de los huevos a partir de la fórmula de la superficie de un elipsoide, así como su densidad.

Se ha determinado el tiempo de eclosión de los huevos sometidos a diferentes temperaturas, para lo que se han utilizado hembras procedentes de las marismas de Villafranco del Guadalquivir que portaban huevos en la primera etapa del desarrollo embrionario. Distintos grupos de hembras se introdujeron en acuarios de 27 litros de capacidad en circuito cerrado, con aireación constante, fotoperíodo de 16: $8 \mathrm{~h}$ (luz-oscuridad) y agua de su lugar de procedencia que se renovaba periódicamente. Un acuario se mantuvo a temperatura ambiente, mientras que a los restantes se les ajustó la temperatura a 25 y $30{ }^{\circ} \mathrm{C}$ mediante la acción de termostatos, conseguiéndose temperaturas medias de $23,3,24,9$ y $29,9^{\circ} \mathrm{C}$ durante el ensayo.

\section{RESULTADOS}

\section{Estadíos de desarrollo}

La Fig. 1 representa diversos momentos del proceso de desarrollo de los huevos durante la incubación, así como el momento de liberación de la zoea 1.

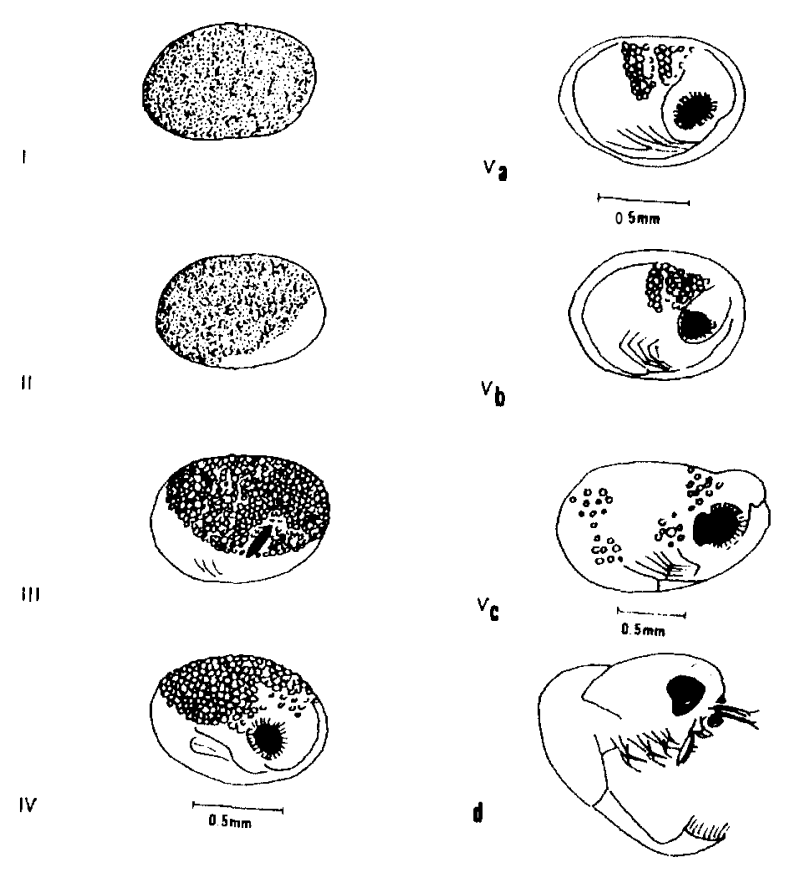

FIGURA 1. Grados de desarrollo de los huevos de Palaemonetes varians: I, II, III, IV (estadios 1 a 4), Va, Vb, Vc (estadio 5) y d (zoea).

FIGURE 1. Developmental stages of Palaemonetes vurians eggs: I, II, III, IV (stages 1 to 4), Va, Vb, Vc (stage 5) y d (zoea).

ESTADÍO 1 (I). Huevos recién fecundados. Sólo se diferencia un vitelo de apariencia homogénea finamente granulado, que comprende el $100 \%$ del volumen del huevo. Su color es ligeramente verdoso, tomando más consistencia cuando se encuentra integrado en el conjunto de la puesta.

ESTADÍO 2 (II). El vitelo tiene una apariencia más granulosa que en el caso anterior y ocupa un volumen algo más reducido, ocupando aproximadamente un $75 \%$ del total del huevo. El embrión aparece como una región transparente sin marcas aparentes. El color del huevo se acerca más a los tonos amarillentos.

ESTADÍO 3 (III). Aparecen las manchas oculares bien definidas, de forma alargada y dispuestas de modo que su eje mayor se dispone oblicuamente con respecto al eje dorsal del huevo, apareciendo rodeadas de gránulos de vitelo de mayor tamaño que en los estadíos anteriores. Estos gránulos ocupan aproximadamente un $50 \%$ del volumen, reduciéndose paulatinamente para dejar espacio al embrión. Se observa débilmente metamerización, existiendo una diferencia patente entre el color amarillento del vitelo y el color blanquecino del embrión.

ESTADÍO 4 (IV). Las manchas oculares presentan un contorno ovalado netamente definido rodeadas de una zona periférica clara. El vitelo ocupa aproximadamente un $25 \%$ del volumen total del huevo y se localiza en la zona dorsal del embrión, en el cual ya se diferencia el abdomen. 
ESTADÍO 5. Se distingue la larva zoea replegada sobre sí misma, con el abdomen en contacto con el rostro ventralmente. Los ojos son muy conspicuos y de gran tamaño. La región cefálica del embrión comienza a definirse. El vitelo queda restringido a la región torácico dorsal, en forma de gránulos bien definidos que quedan unidos en pequeños grupos ( $\mathrm{Va}$ y $\mathrm{Vb}$ ). En el momento previo a la eclosión se manifiesta una elongación del huevo en dirección cefalocaudal; la región cefálica produce una hendidura en la superficie del huevo y se pierde la forma típica del mismo. Algunos gránulos de vitelo individualizados emigran hacia la región ventral para ser liberados en el momento de la eclosión, la cual se produce con el despliegue del abdomen (Vc y d).

\section{Biometría de los huevos}

En la Tabla 1 se muestra los parámetros biométricos de los huevos en función de los grados de desarrollo, procedentes de Ayamonte y Villafranco del Guadalquivir respectivamente. Los valores medios aumentan progresivamente en función del estadío de desarrollo, salvo en el caso del peso. Las características de las hembras ovígeras aparecen en la Tabla 2.

La evolución de las medidas biométricas (valores medios) de las dos series de huevos con respecto al eje mayor de los mismos se representa en la Fig. 2. En ambos casos existe un aumento del eje menor, relación $\mathrm{L} / \mathrm{l}$, volumen y superficie, y una disminución de la densidad y de la relación superficie/volumen. La comparación de las dos series de medidas de volumen de los huevos muestra valores similares para las tallas de ambas series (t-test, $\mathrm{p}<0,001$ ), al igual que en el caso de los ejes mayor y menor.

El esfuerzo reproductivo, expresado como la cantidad relativa de peso de huevos por puesta (peso de la puesta/peso de la hembra ovígera) (MASHTKO, 1983), ha resultado ser 1,43 veces mayor en la población de Villafranco del Guadalquivir (t-test, $\mathrm{p}<0,001$ ), con un valor medio (ISD) de 17,59 $\pm 2,45$ frente al de 12,23 $\pm 3,88$ de Ayamonte.

\section{Incubación de huevos y supervivencia del progenitor}

Los huevos de las hembras mantenidas a temperatura ambiental tardaron 15,5 días de media en completar su desarrollo embrionario y eclosionar. Los huevos sometidos a $24,9{ }^{\circ} \mathrm{C}$ tardaron 14 días y los de $29,9^{\circ} \mathrm{C}, 12,3$ días de media. En la Fig. 3 se muestra la supervivencia de las hembras en cada ensayo. La totalidad de las hembras sometidas a mayor temperatura murieron tras eclosionar su puesta en un período máximo de un día. Esto mismo se observó a la temperatura intermedia, pero
TABLA 1.- Biometna de los huevos de Palaemonetes varians (A: Ayamonte. B: Villafranco) en función del grado de desarrollo de los mismos.

TABLE 1.-Biometry of Palaemonetes varians eggs (A: Ayamonte. B: Villafranco) according to their developmental stage.

\begin{tabular}{lcccccc}
\multicolumn{7}{c}{ A: Ayámonte } \\
\cline { 2 - 6 } ESTADÍO & I & II & III & IV & Va,b & Vc \\
\cline { 2 - 6 } \multicolumn{1}{c}{ n } & 30 & 30 & 30 & 30 & 30 & 30 \\
EJE MAYOR (mm) & & & & & \\
Media & 0,910 & 0,946 & 1,030 & 1,098 & 1,196 & 1,555 \\
S.D.0,049 & 0,046 & 0,055 & 0,038 & 0,029 & 0,081 & \\
Mínimo & 0,80 & 0,80 & 0,90 & 1,00 & 1,10 & 1,40 \\
Máximo & 1,00 & 1,00 & 1,10 & 1,20 & 1,30 & 1,70 \\
EJE MENOR (mm) & & & & & & \\
Media & 0,683 & 0,716 & 0,793 & 0,818 & 0,816 & 0,851 \\
S.D. & 0,047 & 0,059 & 0,021 & 0,038 & 0,035 & 0,049 \\
Mínimo & 0,60 & 0,60 & 0,70 & 0,75 & 0,80 & 0,80 \\
Máximo & 0,80 & 0,90 & 0,80 & 0,90 & 0,90 & 0,90 \\
VOLUMEN(ml) & & & & & & \\
Media & 0,223 & 0,247 & 0,339 & 0,386 & 0,418 & 0,593 \\
S.D. & 0,034 & 0,055 & 0,025 & 0,041 & 0,039 & 0,081 \\
Mínimo & 0,15 & 0,16 & 0,27 & 0,32 & 0,36 & 0,46 \\
Máximo & 0.33 & 0,42 & 0,36 & 0,50 & 0,50 & 0,72 \\
PESO(mg) & & & & & & \\
Media & 0,492 & 0,430 & 0,428 & 0,576 & 0,434 & 0,785 \\
S.D. & 0,142 & 0,154 & 0,106 & 0,120 & 0,163 & 0,221 \\
Mínimo & 0,36 & 0,25 & 0,33 & 0,43 & 0,27 & 0,63 \\
Máximo & 0,64 & 0,53 & 0,54 & 0,66 & 0,60 & 1,04 \\
\hline & & & & & & \\
\hline
\end{tabular}

\begin{tabular}{|c|c|c|c|c|c|c|}
\hline \multicolumn{7}{|c|}{ B: Villafranco } \\
\hline ESTADÍO & I & II & III & IV & $\mathrm{Va}, \mathrm{b}$ & $\mathrm{Vc}$ \\
\hline $\mathrm{n}$ & 30 & 30 & 30 & 30 & 30 & 23 \\
\hline \multicolumn{7}{|c|}{ EJE MAYOR (mm) } \\
\hline Media & 0,866 & 0,901 & 0,963 & 1,050 & 1,090 & 1,410 \\
\hline S.D. & 0,046 & 0,044 & 0,066 & 0,055 & 0,080 & 0,146 \\
\hline Mínimo & 0,80 & 0,80 & 0,80 & 0,95 & 0,95 & 1,30 \\
\hline Máximo & 0,95 & 1,00 & 1,10 & 1,20 & 1,20 & 1,70 \\
\hline \multicolumn{7}{|c|}{ EJE MENOR (mm) } \\
\hline Media & 0,731 & 0,756 & 0,783 & 0,781 & 0,843 & 0,843 \\
\hline S.D. & 0,042 & 0,043 & 0,040 & 0,035 & 0,052 & 0,058 \\
\hline Mínimo & 0,65 & 0,65 & 0,70 & 0,70 & 0,75 & 0,80 \\
\hline Máximo & 0,80 & 0,80 & 0,85 & 0,85 & 0,90 & 1,00 \\
\hline \multicolumn{7}{|c|}{$\operatorname{VOLUMEN}(\mathrm{ml})$} \\
\hline Media & 0,243 & 0,271 & 0,310 & 0,336 & 0,410 & 0,527 \\
\hline S.D. & 0,030 & 0,032 & 0,041 & 0,035 & 0,074 & 0,092 \\
\hline Mínimo & 0,19 & 0,19 & 0,23 & 0,28 & 0,29 & 0,43 \\
\hline Máximo & 0,30 & 0,31 & 0,37 & 0,41 & 0,50 & 0,72 \\
\hline \multicolumn{7}{|l|}{ PESO (mg) } \\
\hline Media & 0,393 & 0,347 & 0,439 & 0,430 & 0,462 & 0,384 \\
\hline S.D. & 0,099 & 0,008 & 0,078 & 0,010 & 0,089 & 0,087 \\
\hline Mínimo & 0,28 & 0,33 & 0,35 & 0,41 & 0,36 & 0,28 \\
\hline Máximo & 0.48 & 0.35 & 0,50 & 0,43 & 0.54 & 0.45 \\
\hline
\end{tabular}




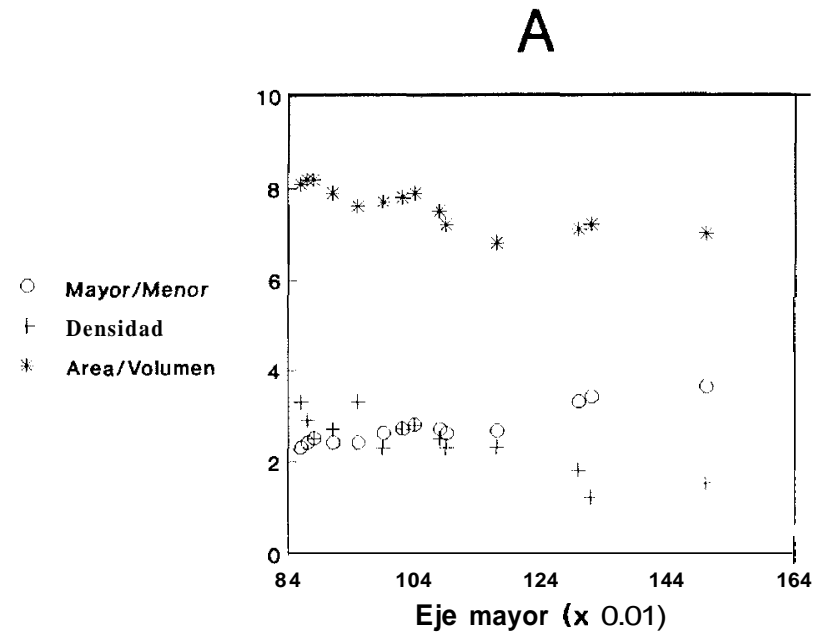

B

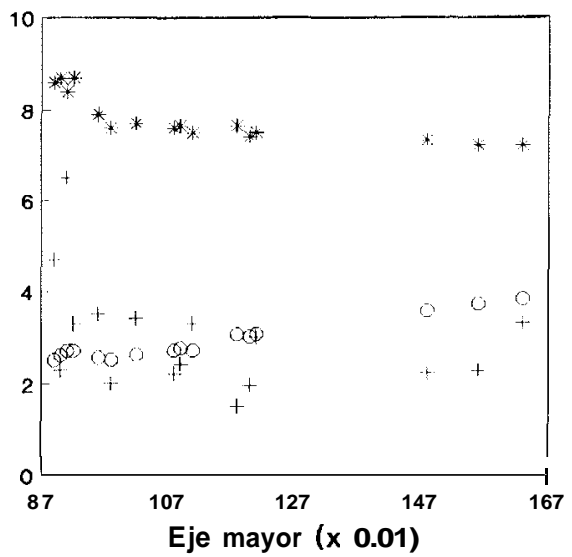

FIGURA 2.- Valores medios de las medidas biométricas de los huevos de Palaemonetes varians en función del crecimiento longitudinal de los mismos. A: Villafranco. B: Ayamonte.

FIGURE 2.- Mean biometric measures of Palaemonetes varians eggs in relation to egg length. A: Villafranco. B: Ayamonte.

después de un período de tiempo mayor, no observándose en ninguna de las hembras sometidas a temperatura ambiente.

\section{DISCUSIÓN Y CONCLUSIONES}

Respecto a las medidas de longitud de los huevos, aparecen mayores cambios en el eje mayor conforme avanza el desarrollo, siendo el crecimiento del huevo principalmente longitudinal, este incremento aumenta notablemente en el último estadío (Fig. 1, Vc), debido a la elongación de la larva previa a la eclosión. La disminución de la relación superficie/volumen y de la densidad
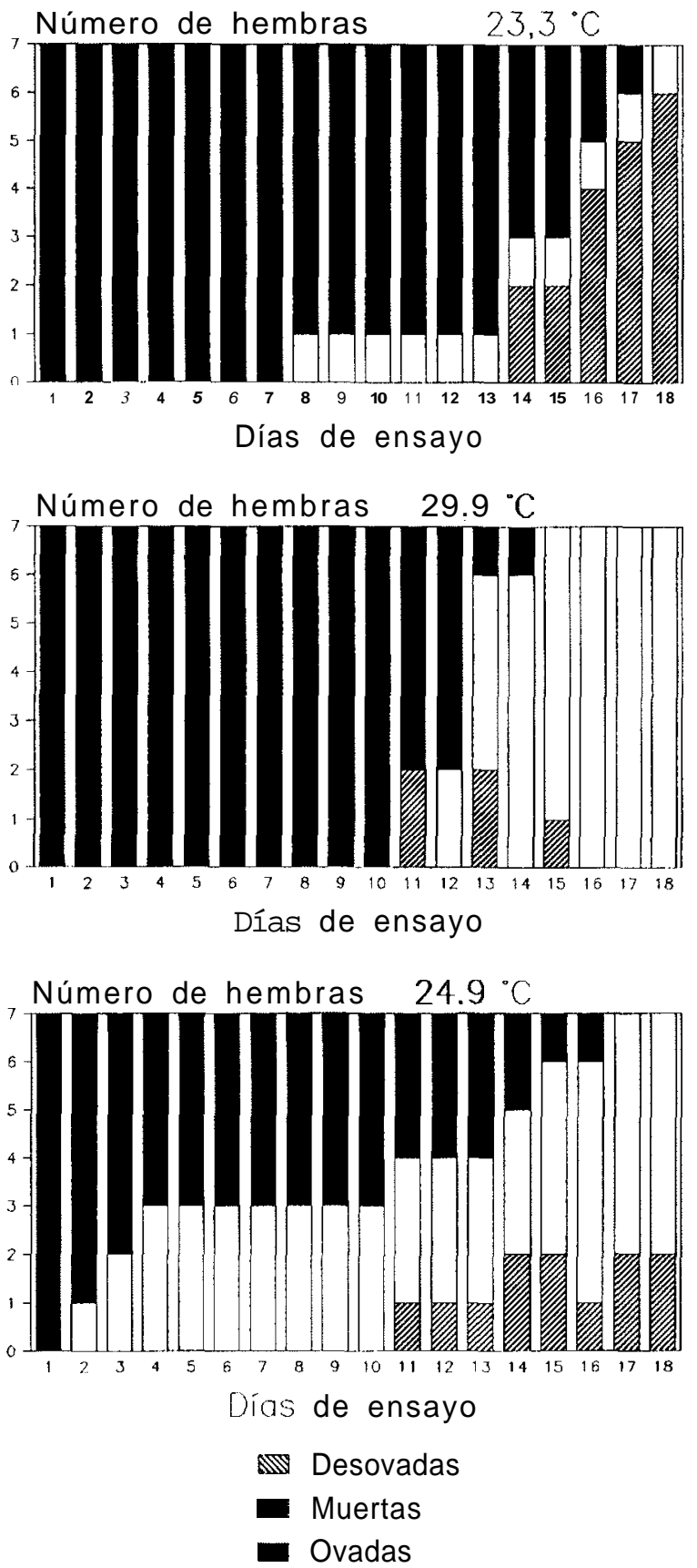

FIGURA 3.- Resultados de los ensayos de eclosión con Palaemonetes varians para las distintas temperaturas y en función del tiempo de incubación. FIGURE 3.- Results of the hatching tests with Palaemonetes varians for different temperatures and different incubation times.

permiten suponer un aumento necesario de la energía invertida en el cuidado de la puesta por parte del progenitor. Para mantener la tasa de intercambio sería necesario aumentar la ventila- 
ción, y el aumento de flotabilidad del huevo exigiría mayor inversión de energía para el mantenimiento de la posición.

TABLA 2.- Biometría de las hembras progenitoras de Palaemonetes varians. TABLE 2.- Biometry of ovigerous females of Palaemonetes vuriuns.

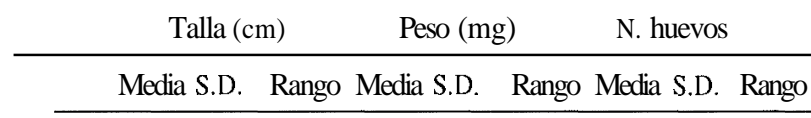

$\begin{array}{llllllllll}\text { SEVILLA } & 3,5 & 0,18 & 3,20-3,90 & 0,68 & 0,11 & 0,44-0,90 & 298 & 64,89 & 162-413\end{array}$ $\begin{array}{llllllllll}\text { HUELVA } & 3,74 & 0,20 & 3,40-4,20 & 0,75 & 0,11 & 0,52-1,02 & 218 & 75,15 & 122-363\end{array}$

SOLLAUD (1938) y HOLTHUIS (1987) citan tallas de huevos de 0,8 mm, mientras que ZARIQUIEY (1968) recoge un valor de $0,51 \mathrm{~mm}$. GURNEY (1924) cita varios intervalos de medida tomando como referencia a Mortensen, Boas y SOLLAUD, siendo solamente los datos correspondientes a la forma del norte $(0,75 \mathrm{~mm})$ (Palaemonetes varians microgenitor BOAS) y los citados por el propio GURNEY (0,9-1,2 mm) los que pertenecen a la especie $P$. varians en el sentido actual. Las medidas de tallas obtenidas son mayores que las citadas anteriormente, lo cuál puede ser debido a que estos autores no especifican el estadío de desarrollo del huevo.

Los resultados obtenidos en la comparación de la biometría de las dos series de huevos. contrastan con los obtenidos para otras especies como P. paucidens y M. nipponense (MASHIKO, 1982 y 1983) entre agua dulce y salobre. En estos, la variación del tamaño del huevo está determinada por un fenómeno de adaptación a las diferencias de energía requerida para el mantenimiento del equilibrio osmótico. En nuestro caso, aunque existen diferencias de salinidad en los dos ambientes, la similitud de las tallas de los huevos parece indicar una preadaptación a ambientes de salinidades fluctuantes, de forma que el huevo está capacitado para soportar los cambios de demanda de energía para la osmoregulación, presentando cantidades de vitelo suficientes para cubrir el desgaste energético cuando se produzcan bajas salinidades.

LE ROUX (1989) encuentra que las larvas de $P$. varians no presentan modificaciones susceptibles de ser interpretadas como reacciones adaptativas frente a diferentes niveles de salinidad. Estos resultados en estadíos larvarios junto con los obtenidos en este trabajo, contrastan con las diferencias encontradas por HELDT (1953).

El concepto de esfuerzo reproductivo es útil en el análisis de la estrategia reproductiva (PIANKA, 1978). De acuerdo con el principio de asignación (CODY, 1966), un esfuerzo reproductivo menor incrementa la capacidad de supervivencia del progenitor. El aumento de la capacidad de supervivencia de la hembra progenitora debe ser una adaptación a las condiciones ina- decuadas del agua dulce, por lo que en M. nipponense se obtienen valores de esfuerzo reproductivo menores en hembras de aguas dulces (MASHIKO, 1983); este rnismo autor no encuentra diferencias en las hembras de P. paucidens de diferentes salinidades, y concluye que este factor ambiental debe ser neutral para el esfuerzo reproductivo de esta especie (MASHIKO, 1982). Las hembras de $P$. pugio presentan una mayor fecundidad como respuesta adaptativa a una mayor presión por predación debido a la competencia de $P$. vulgaris y sin embargo, ambas especies no presentan diferencias en cuanto a esfuerzo reproductivo (YAN, 1987).

$P$. varians presenta en este caso, una respuesta adaptativa contraria a la observada para M. nipponense, el esfuerzo reproductivo de las hembras a menor salinidad es mayor, desplazando la capacidad de supervivencia desde el progenitor hacia la puesta. Para mantener un tamaño de huevo óptimo a bajas y fluctuantes salinidades, P. varians sigue una estrategia adaptativa "aseguradora" de la puesta, basada en un mayor gasto energético en la reproducción.

El tiempo de incubación de los huevos ha sido inversamente proporcional a la temperatura. Es de destacar la poca variación en los tiempos de desarrollo embrionario entre las temperaturas ensayadas. En otros Palaemónidos como Palaemon serratus, se obtienen mayores diferencias en el período de incubación para temperaturas próximas (RAMONELL et al., 1987), si bien estas son inferiores a las nuestras.

Aunque las hembras utilizadas para estas experiencias no han sido aclimatadas previamente , la rápida mortalidad observada en las hembras mantenidas a $30{ }^{\circ} \mathrm{C}$ puede ser importante por su influencia en la dinámica de las poblaciones silvestres, debido a las altas temperaturas que se alcanzan en los meses de verano en nuestra área geográfica. Hembras de una experiencia anterior sometidas a una temperatura de 28 "C, morían tras un período medio de 2,5 días una vez ocurrida la eclosión. En el caso de las hembras del acuario a $24,9{ }^{\circ} \mathrm{C}$, debido a que las muertes se han producido en tiempos muy variables tras la eclosión y a que son pocos ejemplares, no es posible establecer una relación directa entre eclosión y muerte de la hembra.

La causa de la mortandad parece ser el estrés reproductivo, por lo que una condición adversa como es una alta temperatura, incide negativamente en la supervivencia de la hembra a favor de la supervivencia de la puesta, hecho que apoya la hipótesis de estrategia adaptativa ya comentada.

\section{BIBLIOGRAFÍA}

ANTHEUNISSE, L. J., J. J. LAMMENS \& N.P. V AN DEN HOVEN 1977. Diurnal activities and tidal migra- 
tions of the brackish water prawn Palaemonetes varians (Leach) (Decapoda, Caridea). Crustaceana, 29(2): 203-217.

CALDENTEY, M. A., I. J. LOZANO, F. HERNANDEZ, J. A. GONZALEZ y J. I. SANTANA 1988. Introducción al estudio de la fecundidad de Plesionika edwardsii (Brant,1851) (Crustacea, Caridea, Pandalidae). Actas VISimp. Iber. Estud. Bentos Mar.

CODY, M. L. 1966. A general theory of clutch size. Evolution, 20: 174-184.

GURNEY, R. 1924. The larval Development of some British Prawns (Palaemonidae). I. Palaemonetes varians. Proc. Zool. Soc. Lond.: 297-328.

HELDT, J. H. 1953. Palaemonetes varians (Leach) (Crustacé, Décapode) au Lac Kelbia. Cas de poecilogonie dans une même nappe d'eau. Bull. Stn océanogr. Salammbô, 44: 1-14.

HOLTHUIS, L. B. in FISCHER, W., M.-L. BAUCHOT et M. SCHNEIDER (rédacteurs) 1987. Fiches FAO D'Identification de espèces pour les besoins de la peche. Mediterranée et mer noire. Zone de Peche 37 (Rév. 1) (I). Végétaux et Invertébrés. FAO (I): 760 pp.

LE ROUX, A. 1989. Contribution a l'etude du developpement larvaire et de la metamorphose chez des Crustaces eucarides. Thèse Université de Rennes I: 373 pp.

MASHIKO, K. 1982. Differences in both the eggs size and the clutch size of the freshwater prawn Palaemon paucidens de Haan in the Sagami river. Japanese Journal of Ecology, 32(4): 445-451.

MASHIKO, K. 1983. Differences in the Eggs and Clutch Sizes of the Prawn Macrobrachium nipponense (de Haan) between Brackish and Fresh Waters of a River. Zoological Magazine, 92: 1-9.

PIANKA, E. R. 1978. Evolutionary ecology, 2nd. ed., HAPER \& ROW, New York. 420 pp.

RAMONELL, R., R. BERMUDEZ y A. LANDIN 1987. Maduración de los huevos de Palaemon serratus (Pennant) e influencia de la temperatura en el tiempo de incubación. Cuad. Marisq. Publ. Téc., 12: 285-290.

SOLLAUD, E. 1938. Sur un Palaemonetes endémique, Palaemonetes zariquieyi, N. SP. Localisé dans la plaine littorale du Golfe de Valence. Trav. Sta. Zool. Wimereux, 13: 635- 645.

YAN, H. Y. 1987. Comparative reproductive strategies of the grass shrimps, Palaemonetes vulgaris and Palaemonetes pugio (Decapode, Natantia) in Great Sippewissett salt marsh, Massachusetts, U.S.A. Crustaceana, 52(2): 141-148.

YUFERA, M. y A. RODRÍGUEZ 1985. Tasa de alimentación y crecimiento de Palaemonetes varians (Crustacea: Palaemonidae) durante el desarrollo larvario. Inv. Pesq., 49(4): 597- 606.

ZARIQUIEY, R. 1968. Crustáceos Decápodos Ibéricos. Inv. Pesq., 32: 510 pp. 